\title{
Aspects of Anisotropic Fractional Quantum Hall Effect in Phosphorene
}

\author{
Areg Ghazaryan and Tapash Chakraborty* \\ Department of Physics and Astronomy, University of Manitoba, Winnipeg, Canada R3T 2N2
}

(Dated: June 22, 2021)

\begin{abstract}
We have analyzed the effects of the anisotropic energy bands of phosphorene on magnetoroton branches for electrons and holes in the two Landau levels close to the band edges. We have found that the fractional quantum Hall effect gap in the lowest (highest) Landau level in conduction (valence) band is slightly larger than that for conventional semiconductor systems and therefore experimentally observable. We also found that the magnetoroton mode for both electrons and holes consists of two branches with two minima due to the anisotropy. Additionally, we show that due to the anisotropy, there is a second mode with positive dispersion, well separated from the magnetoroton mode for small wave vectors. These novel features of the collective mode can be observed in resonant inelastic light scattering experiments.
\end{abstract}

With the advent of graphene [1, 2] the two dimensional (2D) materials have gained renewed attention during the last decade because of their remarkable electronic, optical and mechanical properties and for potential device applications. Practical application of graphene in electronic devices is however limited due to its zero band gap. Although there are external means to create band gap in graphene, such as application of a bias voltage to bilayer graphene, in the past few years intense search has been undertaken to find other 2D materials, which in addition to exhibiting mobilities close to graphene, also posses a considerable band gap in their normal state. There were several Dirac materials, such as silicene [3], germanene [4], $\mathrm{MoS}_{2}$ and other group-VI transition-metal dichalcogenides [5] that have been lately explored and promising results were observed.

In the past year another material which has gained popularity in this context, is the $2 \mathrm{D}$ version of black phosphorus (BP) [6-10], the single layer of BP known as phosphorene. BP is the most stable allotrope of Phosphorus at room temperature and pressure. Few-layer BP can be obtained through mechanical exfoliation method akin to graphene. However, unlike graphene the BP layers are not perfectly flat. Due to the $s p^{3}$ hybridization of $3 s$ and $3 p$ atomic orbitals the layers of BP form a puckered surface. The band gap of BP depends on the number of layers of the sample, and it ranges from $0.3 \mathrm{eV}$ in the bulk case at the $\mathrm{Z}$ point in the Brillouin zone to 1.5 - 2 $\mathrm{eV}$ for few layer and monolayer case at the $\Gamma$ point. The mobilities of the few-layer BP obtained so far lie in the range of $1000-5000 \mathrm{~cm}^{2} \mathrm{~V}^{-1} \mathrm{~s}^{-1}$ [6, 11, 12]. Considerable progress has been made in the study of the electronic and optical properties of this material both experimentally [6 8, 11 13] and theoretically [14 24]. Higher mobilities were achieved by sandwiching the BP in hexagonal boron nitride flakes and placing on top of the graphite back gate that has enabled observation of the integer quantum Hall effect [12]. Clearly the next step in the experimental progress is to reach the regime where the fractional

*Tapash.Chakraborty@umanitoba.ca quantum Hall effect (FQHE) [25] can be observed.

The FQHE in Dirac materials shows many interesting features not found in conventional semiconductor quantum heterostructures. The FQHE states in monolayer and bilayer graphene were investigated theoretically 26 29] and experimentally 30, 31]. It was shown that while the FQHE states in graphene in the $n=0$ Landau level (LL) are completely identical to the case of conventional semiconductor systems, graphene exhibits more robust FQHE states in the $n=1$ LL with a bigger gap than in the $n=0 \mathrm{LL}$ 27]. That behavior is not present in conventional semiconductor systems. In bilayer graphene the application of a bias voltage results in some LLs the observation of the phase transition between several incompressible FQHE and compressible phases 28, 31]. The FQHE in silicene and germanene indicated that because of the strong spin-orbit interaction present in these materials as compared to graphene, the electron-electron interaction and the FQHE gap are significantly modified [32]. The puckered structure of phosphorene exhibits a lower symmetry than graphene. This results in anisotropic energy spectra and other physical characteristics of phosphorene, both in momentum and real space in the 2D plane 9, 10]. Therefore it is desirable to explore how this anisotropy of phosphorene manifests itself in the FQHE states.

In this work we consider the FQHE states in phosphorene for filling factor $\nu=1 / 3$ in the conduction band and $\nu=2 / 3$ in the valence band in two LLs closest to the band edges. We analyze the energy spectra and the magnetoroton modes of the system with finite number of electrons using the exact diagonalization scheme. We observe the FQHE gap in the lowest (highest) LL in the conduction (valence) band, with the gap being slightly higher than in conventional semiconductor systems. This indicates that the FQHE should be observable in phosphorene. We also show that both the gap of the FQHE and the magnetoroton mode are similar for the lowest LL in the conduction band and highest LL in the valence band. The anisotropy of phosphorene manifests itself in two different ways. We find that the magnetoroton mode in phosphorene (for electrons and holes) is split into two branches with two minima due to the 
anisotropy. We also find that the anisotropy causes a second mode with positive dispersion to appear that is well separated from the magnetoroton mode for small wave vectors and is therefore experimentally more accessible than for isotropic systems. For the FQHE states in the second LLs of the conduction and valence band of phosphorene, the FQHE gap is an order of magnitude smaller than for the lowest LLs and approaches a soft mode, with possible incompressible to compressible phase transition.

In what follows we consider a finite-size system of electrons in 2D phosphorene in the toroidal geometry using the exact diagonalization procedure [25]. The manybody Hamiltonian of the system is

$$
\mathcal{H}=\sum_{i}^{N_{e}} \mathcal{H}_{\mathrm{P}}^{i}+\frac{1}{2} \sum_{i \neq j}^{N_{e}} V_{i j}
$$

where $\mathcal{H}_{\mathrm{P}}$ is the single-particle Hamiltonian in phosphorene and the second term is the Coulomb interaction. Since we are interested in the FQHE in the low lying LL, which are situated close to the conduction and the valence band edges, we employ the continuum approximation of the single-particle Hamiltonian derived from the microscopic tight-binding approach [19]. In all our calculations we take the $x$ axis along the armchair direction and the $y$ axis along the zigzag direction. Then the single-particle Hamiltonian in single-layer phosphorene in the continuum approximation has the form [19]

$$
\begin{aligned}
\mathcal{H}_{\mathrm{P}}(\mathbf{k}) & =\left(\begin{array}{cc}
H_{0}(\mathbf{k}) & H_{1}(\mathbf{k}) \\
H_{1}^{*}(\mathbf{k}) & H_{0}(\mathbf{k})
\end{array}\right) \\
H_{0}(\mathbf{k}) & =u_{0}+\eta_{x} k_{x}^{2}+\eta_{y} k_{y}^{2} \\
H_{1}(\mathbf{k}) & =\delta+\gamma_{x} k_{x}^{2}+\gamma_{y} k_{y}^{2}+i \chi k_{x}
\end{aligned}
$$

where $\mathbf{k}$ is the wave vector and all parameters in $H_{0}(\mathbf{k})$ and $H_{1}(\mathbf{k})$ are related to five hopping amplitudes in the tight-binding model [19]: $u_{0}=-420 \mathrm{meV}, \eta_{x}=$ $10.1 \mathrm{meV} \cdot \mathrm{nm}^{2}, \eta_{y}=5.8 \mathrm{meV} \cdot \mathrm{nm}^{2}, \delta=760 \mathrm{meV}, \gamma_{x}=$ $38.3 \mathrm{meV} \cdot \mathrm{nm}^{2}, \gamma_{y}=39.3 \mathrm{meV} \cdot \mathrm{nm}^{2}, \chi=525 \mathrm{meV} \cdot \mathrm{nm}$. For these parameters the band edges of the conduction and the valence band are located at the energies $E_{c}=340 \mathrm{meV}$ and $E_{v}=-1180 \mathrm{meV}$, with energy gap $E_{g}=1520 \mathrm{meV}$. In a magnetic field, we first write $\mathbf{k}=-i \nabla+e \mathbf{A} / \hbar c$, where the vector potential is in the Landau gauge $\mathbf{A}=B x \hat{\mathbf{y}}$. For all the FQHE states considered here the many-body ground state is fully spin polarized. We henceforth disregard both the electron spin and the Zeeman energy. For the next step we cast the Hamiltonian (2) into the second quantized form using the ladder operator $a=\frac{1}{\sqrt{\hbar \omega_{c}}}\left[\sqrt{\eta_{x}} k_{x}-i \sqrt{\eta_{y}} k_{y}\right]$, where $\hbar \omega_{c}=2 \sqrt{\eta_{x} \eta_{y}} / \ell_{0}^{2}$ and $\ell_{0}=\sqrt{\hbar c / e B}$ is the magnetic length. It is easy to verify that $\left[a, a^{\dagger}\right]=1$. Then the components of the Hamiltonian (2) are

$$
\begin{aligned}
H_{0} & =u_{0}+\hbar \omega_{c}\left(a^{\dagger} a+\frac{1}{2}\right), \\
H_{1} & =\delta+\hbar \omega_{c}\left(s\left(a^{\dagger} a+\frac{1}{2}\right)+\frac{1}{4}(s-t)\left(a^{\dagger}-a\right)^{2}\right) \\
& +i \hbar \omega_{c} \tilde{\chi}\left(a^{\dagger}+a\right),
\end{aligned}
$$

where $s=\gamma_{x} / \eta_{x}, t=\gamma_{y} / \eta_{y}, \tilde{\chi}=\chi / 2 \sqrt{\eta_{x} \hbar \omega_{c}}$. We search for the eigenvalues of the Hamiltonian (2) in the form

$$
\psi(x, y)=\left(\begin{array}{c}
\sum_{n=0}^{n_{\max }} A_{n} \phi_{n, k_{y}}(x, y) \\
\sum_{n=0}^{n_{\max }} B_{n} \phi_{n, k_{y}}(x, y)
\end{array}\right),
$$

where $A_{n}$ and $B_{n}$ are constants and $\phi_{n, k_{y}}(x, y)$ are the usual harmonic oscillator wave functions

$$
\begin{aligned}
\phi_{n, k_{y}}(x, y)=\frac{\alpha^{\frac{1}{4}}}{\sqrt{2^{n} n ! L_{y} \sqrt{\pi}}} & e^{i k_{y} y} e^{-\frac{\alpha\left(x+k_{y} \ell_{0}^{2}\right)^{2}}{2}} \\
& \times H_{n}\left(\sqrt{\alpha}\left(x+k_{y} \ell_{0}^{2}\right)\right),
\end{aligned}
$$

where $\alpha=\hbar \omega_{c} / 2 \eta_{x}, L_{y}$ is the sample size in the $y$ direction and $k_{y}$ is the wave vector in the $y$ direction, which is conserved in the chosen gauge. Diagonalizing the Hamiltonian matrix obtained with the wave function (3) we determine the eigenvalues of the Hamiltonian (2), which are the LLs of phosphorene and the appropriate wave functions by determining the constants $A_{n}$ and $B_{n}$.

In order to consider the many-body states we proceed in the same way as for conventional semiconductor systems [25, 33]. We consider the many-body states in the system in a toroidal geometry, i.e., we apply the periodic boundary conditions (PBC) on both directions of the system [25, 33, 34] and cast the wave functions (3) and (4) into the PBC preserving form. This process naturally defines the magnetic translation unit cell, which we take rectangular in our calculations with the sides $L_{x}$ and $L_{y}$, $L_{x} L_{y}=2 \pi \ell_{0}^{2} N_{s}$, where $N_{s}$ is the number of magnetic flux quanta passing through the unit cell and is an integer number. $N_{s}$ also characterizes the degeneracy of each LL. We take $N_{e}=p N$ and $N_{s}=q N$, where $N_{e}$ is the number of electrons, $N$ is the greatest common divisor of $N_{e}$ and $N_{s}, p$ and $q$ are integers. The filling factor in a LL is defined as $\nu=p / q$. We then construct the many-body states from the appropriate single-particle states and calculate the Hamiltonian matrix of the form (10) with this many-body basis states. We make use of the many-body translational symmetry to reduce the size of the Hilbert space [25, 33]: We define the relative translation operator $T_{i}^{\mathrm{R}}(\mathbf{a})$ and note that the translations $T_{i}^{\mathrm{R}}\left(p \mathbf{L}_{m n}\right)$ preserve the Hilbert space and commute with the Hamiltonian (1) and with each other. Here $\mathbf{L}_{m n}=m \mathbf{L}_{x}+n \mathbf{L}_{y}$ is the magnetic translations lattice vector, $\mathbf{L}_{x}$ and $\mathbf{L}_{y}$ are the unit vectors of magnetic translations unit cell, $m$ and $n$ are integers. Therefore we use these relative translation operator $\left(T_{i}^{\mathrm{R}}\left(p \mathbf{L}_{m n}\right)\right.$ eigenstates to bring the complete Hamiltonian matrix into the block-diagonal form 


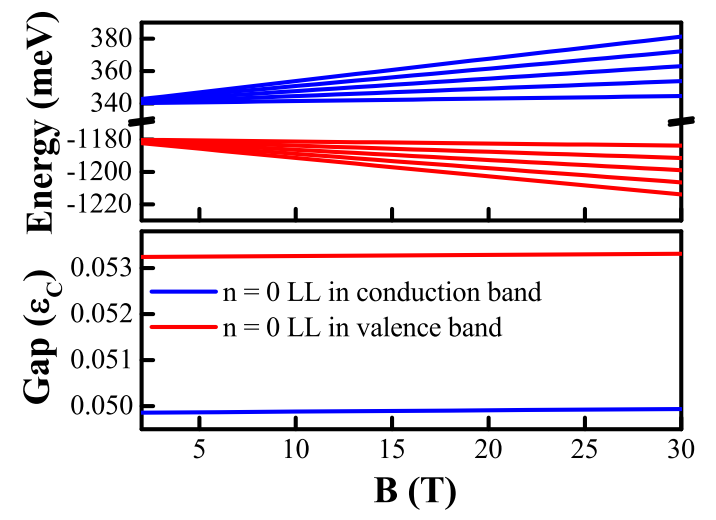

FIG. 1: (a) Magnetic field dependence of few low lying LLs in phosphorene, both in the conduction (blue) and the valence (red) band. (b) Magnetic field dependence of the $\nu=1 / 3$ gap in the conduction band and the $\nu=2 / 3$ gap in the valence band lowest LLs of phosphorene. The finite-size system considered for FQHE gap has six electrons.

and also to characterize each of the many-body states with its appropriate relative momentum $\mathbf{k}_{\mathrm{R}}$.

In Fig. 1 (a) the magnetic field dependence of the few low lying LLs both in the conduction band and in the valence band of phosphorene is shown. The dependence is almost linear and is similar to the case of conventional semiconductors. In fact as was shown previously by several authors [16, 18 20] in the low energy regime the energy bands of the conduction and the valence band can be approximated by parabolic dispersion with anisotropic masses in the $x$ and $y$ directions. Therefore for low-lying LLs and for magnetic fields up to $\sim 25 \mathrm{~T}$ the dependence of the LLs on the magnetic field is linear. For this reason, in considering the FQHE states in the low-lying LLs we can disregard the LL coupling (the possibility for electrons to occupy states in higher LL). In case of systems with parabolic dispersion the LL mixing parameter $\kappa$, the ratio between the Coulomb interaction energy and the cyclotron energy has the $\propto 1 / \sqrt{B}$ dependence [35]. Therefore for high magnetic fields $\kappa<1$ and LL mixing can be safely ignored in our work.

In Fig. 1 (b) the magnetic field dependence of the FQHE magnetoroton gap is presented for the lowest LL in the conduction and in highest LL in the valence band. Due to the particle-hole symmetry the filing factor $\nu=1 / 3$ of electrons in the valence band corresponds to $\nu=2 / 3$ filling for holes. The results are presented for the finite-size system with $N_{e}=6$. The aspect ratio of the magnetic unit cell $\lambda=L_{x} / L_{y}$ is $\lambda=N_{e} / 4$ in all calculations of the FQHE states. As the gap is presented in units of $\epsilon_{\mathrm{C}}=e^{2} / \epsilon \ell_{0}$, where $\epsilon=10.2$ is the dielectric constant, this dependence is due to the mixing of each LL in phosphorene in terms of the harmonic oscillator wave functions (4). In conventional semiconductors the FQHE gap in units of $\epsilon_{\mathrm{C}}$ does not depend on the magnetic field and for a similar system size the gap is 0.041 . Therefore as seen in Fig. 1 (b) the FQHE gaps in phosphorene are

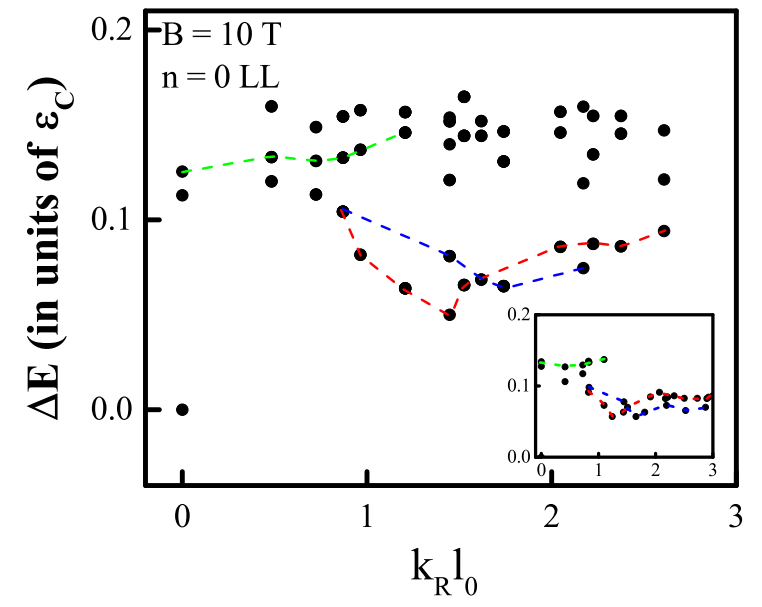

FIG. 2: The energy spectrum of the six-electron FQHE system in phosphorene with $\nu=\frac{1}{3}$ in the lowest LL of the conduction band. The red and blue dash lines show schematically the two branches of the magnetoroton mode. Green dash line shows the second mode described in the text. Inset: the magnetoroton mode and the second mode for $N_{e}=7$.

slightly larger than in conventional systems. The FQHE gap for the LL in the valence band is in fact larger than the gap in the conduction band, but overall all these gaps fall within the same energy range. The dependence of the FQHE gaps on the magnetic field is linear, albeit barely discernable, which again indicates that up to $25 \mathrm{~T}$ the non-trivial character of the LLs in phosphorene compared to conventional systems with parabolic dispersion is less important. The main features of phosphorene that can distinguish it from conventional semiconductor systems in the context of FQHE are the anisotropic nature of its bands, which we address next.

Figure 2 shows the energy spectrum of the six-electron FQHE system in the lowest LL of the conduction band for phosphorene with filling factor $\nu=1 / 3$. The inset shows the low energy collective modes for a seven electron system. As in conventional semiconductor systems, the magnetoroton mode can be clearly identified. The effect of anisotropy of the energy bands of phosphorene is that the magnetoroton mode is split into two branches with two minima. These are schematically shown in the figure with red and blue dashed lines. These kind of branches were actually noted previously in the context of adding a band mass tensor (Galilean metric) to the Hamiltonian in order to investigate the correlation between external and intrinsic metrics of the system 36 38]. The intrinsic metric was treated as a variational parameter which characterizes the shape of the correlation function of the Laughlin state and is obtained by finding the maximum overlap of the exact wave function and the family of Laughlin wave functions parametrized by the intrinsic metric. The splitting of the magnetoroton mode into two branches is possible in graphene in the case of anisotropic electron-electron interaction [39]. The appearance of two branches can be attributed to the 


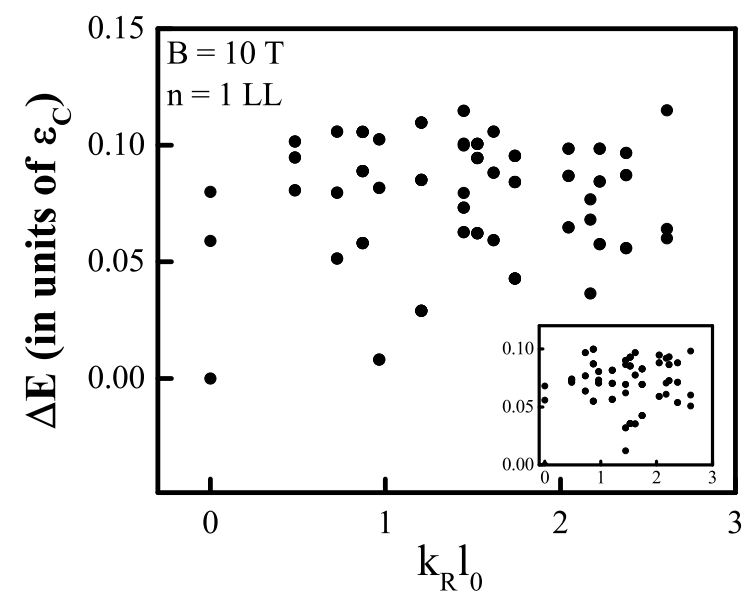

FIG. 3: The energy spectrum of the six-electron FQHE system with $\nu=\frac{7}{3}$ in the second LL of conduction band for phosphorene. Inset: the same spectrum but for a conventional semiconductor system (such as GaAs).

fact that in the anisotropic system the minimum of the magnetoroton mode appears in different $\left|k_{R}\right|$ for different direction of the relative momentum $\mathbf{k}_{\mathbf{R}}$. From this consideration we can conclude that phosphorene presents a real anisotropic system where the ideas of correlation of the external metric (mass tensor in this case) and the intrinsic metric can be explored.

Interestingly, in Fig. 2 we also observe a second mode (schematically shown by a green dashed line) for small wave vectors. It has positive dispersion and merges with the continuum for wave vectors $k_{\mathrm{R}} \ell_{0}>1$. This mode was observed previously in an experiment on the GaAs sample and was attributed to a two-roton state [40]. One possible theoretical explanation for the appearance of the second mode was that two types of shear forces exerted on the liquid state have different direction for different modes [41]. While for isotropic systems these two modes should converge to the same point at $k_{\mathrm{R}} \rightarrow 0$ our studies indicate that for an anisotropic system these two modes are clearly separated for small wave vectors and do not converge to the same point as $k_{\mathrm{R}} \rightarrow 0$. Therefore phosphorene can greatly simplify the identification of the second mode from the magnetoroton mode in resonant inelastic light scattering experiment. It should be noted that possibly anisotropy has played some role in the identification of the second mode even in the original experiment [40]. In that experiment the applied magnetic field is not perpendicular to the sample. The effect of the tilted magnetic field has been exhaustively studied in the experiments on FQHE and it was shown that tilting results in anisotropic transport properties of the sample [42]. The effect of the tilted field on the FQHE states was also addressed theoretically by several authors [43, 44] and it was found that inclusion of the tilted field is similar to adding an anisotropic metric in the system, although for the tilted magnetic field the dynamics is more complicated than simply the inclusion of the anisotropic mass. Therefore, due to the tilted field the system perhaps acquire some sort of anisotropy in the experiment [40], which reveals the second mode. Finally, we have performed similar studies for the highest LL in the valence band for $\nu=2 / 3$ and obtained very similar results.

In Fig. 3 we present the energy spectrum of the sixelectron FQHE system in the second LL of the conduction band for phosphorene with filling factor $\nu=7 / 3$. The same result for the conventional semiconductor system (such as GaAs) is shown as inset. It seems that the two branches of the magnetoroton mode and the second mode are still present. The gaps are however, very small and the system seems to be approaching a soft mode, resulting in a phase transition from the incompressible to a compressible phase. Similar results are also found for the second LL in the valence band.

To summarize, we have studied the FQHE states in phosphorene in two LLs close to the band edges in the conduction and valence band. We have shown that the gaps for $\nu=1 / 3$ of electrons in the lowest LL of the conduction band and $\nu=2 / 3$ for holes in the highest LL of the valence band are slightly larger than for conventional semiconductor systems. Therefore the FQHE states should certainly be observable in phosphorene. The anisotropic band structure of phosphorene causes splitting of the magnetoroton mode into two branches with two minima. For small wave vectors, there is also a second mode with positive dispersion that is clearly separated from the magnetoroton mode. These features should be observable in inelastic light scattering experiments.

The work has been supported by the Canada Research Chairs Program of the Government of Canada.
[1] H. Aoki and M.S. Dresselhaus (Eds.), Physics of Graphene (Springer, New York 2014).

[2] D.S.L. Abergel, V. Apalkov, J. Berashevich, K. Ziegler, and T. Chakraborty, Adv. Phys. 59, 261 (2010).

[3] P. De Padova, C. Ottaviani, C. Quaresima, B. Olivieri, P. Imperatori, E. Salomon, T. Angot, L. Quagliano, C. Romano, A. Vona, M. Muniz-Miranda, A. Generosi, B. Paci and G. Le Lay, 2D Materials 1, 021003 (2014); J. Sone, T. Yamagami, Y. Aoki, K. Nakatsuji and H. Hirayama,
New J. Phys. 16, 095004 (2014).

[4] M.E. Dávila, L. Xian, S. Cahangirov, A. Rubio and G. Le Lay, New J. Phys. 16, 095002 (2014).

[5] Q.H. Wang, K. Kalantar-Zadeh, A. Kis, J. N. Coleman and M. S. Strano, Nature Nanotech. 7, 699712 (2012).

[6] L. Li, Y. Yu, G.J. Ye, Q. Ge, X. Ou, H. Wu, D. Feng, X.H. Chen and Y. Zhang, Nature Nanotech. 9, 372 (2014).

[7] H. Liu, A.T. Neal, Z. Zhu, Z. Luo, X. Xu, D. Tománek and P.D. Ye, ACS Nano 8, 4033 (2014). 
[8] F. Xia, H. Wang and Y. Jia, Nature Commun. 5, 4458 (2014).

[9] L. Kou, C. Chen and S.C. Smith, J. Phys. Chem. Lett. 6, 2794 (2015).

[10] H. Liu, Y. Du, Y. Deng and P.D. Ye, Chem. Soc. Rev. 44, 2732 (2015).

[11] L. Li, G.J. Ye, V. Tran, R. Fei, G. Chen, H. Wang, J. Wang, K. Watanabe, T. Taniguchi, L. Yang, X.H. Chen and Y. Zhang, Nature Nanotech. 10, 608 (2015).

[12] L. Li, F. Yang, G.J. Ye, Z. Zhang, K. Watanabe, T. Taniguchi, Y. Wang, X.H. Chen and Y. Zhang, arXiv:1504.07155 (2015).

[13] S.P. Koenig, R.A. Doganov, H. Schmidt, A.H. Castro Neto and B. Özyilmaz, Appl. Phys. Lett. 104, 103106 (2014).

[14] A.S. Rodin, A. Carvalho and A.H. Castro Neto, Phys. Rev. Lett. 112, 176801 (2014).

[15] T. Low, A.S. Rodin, A. Carvalho, Y. Jiang, H. Wang, F. Xia and A.H. Castro Neto, Phys. Rev. B 90, 075434 (2014).

[16] Y. Jiang, R. Roldán, F. Guinea and T. Low, arXiv:1505.00175.

[17] A.N. Rudenko and M.I. Katsnelson, Phys Rev. B 89, 201408 (2014).

[18] S. Yuan, A.N. Rudenko and M.I. Katsnelson, Phys. Rev. B 91, 115436 (2015).

[19] J.M. Pereira Jr. and M.I. Katsnelson, arXiv:1504.02452

[20] X.Y. Zhou, R. Zhang, Y.L. Zou, D. Zhang, W.K. Lou, F. Cheng, G.H. Zhou, F. Zhai and K. Chang, arXiv:1411.4275

[21] Q. Liu, X. Zhang, L.B. Abdalla, A. Fazzio and A. Zunger, Nano Lett. 15, 1222 (2015).

[22] M. Ezawa, New J. Phys. 16, 115004 (2014).

[23] M. Tahir, P. Vasilopoulos and F.M. Peeters, arXiv:1505.06780

[24] D. Çakir, C. Sevik and F.M. Peeters, arXiv:1506.04707,

[25] T. Chakraborty and P. Pietiläinen, The Quantum Hall Effects (Springer, New York, 1995); The Fractional Quantum Hall Effect (Springer, New York, 1988).

[26] T. Chakraborty and V. Apalkov, in [1] Ch. 8; T. Chakraborty and V.M. Apalkov, Solid State Commun. 175, 123 (2013).

[27] V.M. Apalkov and T. Chakraborty, Phys. Rev. Lett. 97, 126801 (2006).

[28] V.M. Apalkov and T. Chakraborty, Phys. Rev. Lett. 105,
$036801(2010)$

[29] V.M. Apalkov and T. Chakraborty, Phys. Rev. Lett. 107, 186803 (2011).

[30] X. Du, I. Skachko, F. Duerr, A. Luican and E.Y. Andrei, Nature 462, 192 (2009); K.I. Bolotin, F. Ghahari, M.D. Shulman, H.L. Stormer and P. Kim, Nature 462, 196 (2009).

[31] P. Maher, L. Wang, Y. Gao, C. Forsythe, T. Taniguchi, K. Watanabe, D. Abanin, Z. Papić, P. Cadden-Zimansky, J. Hone, P. Kim and C.R. Dean, Science 345, 61 (2014).

[32] V.M. Apalkov and T. Chakraborty, Phys. Rev. B 90, 245108 (2014).

[33] F.D.M. Haldane, Phys. Rev. Lett. 55, 2095 (1985).

[34] T. Chakraborty, Surf. Sci. 229, 16 (1990); Adv. Phys. 49, 959 (2000); T. Chakraborty and P. Pietiläinen, Phys. Rev. Lett. 76, 4018 (1996); T. Chakraborty and P. Pietiläinen, Phys. Rev. Lett. 83, 5559 (1999); T. Chakraborty and P. Pietiläinen, Phys. Rev. B 39, 7971 (1989); V.M. Apalkov, T. Chakraborty, P. Pietiläinen, and K. Niemelä, Phys. Rev. Lett. 86, 1311 (2001); T. Chakraborty, P. Pietiläinen, and F.C. Zhang, Phys. Rev. Lett. 57, 130 (1986); T. Chakraborty and F.C. Zhang, Phys. Rev. B 29, 7032 (R) (1984); F.C. Zhang and T. Chakraborty, Phys. Rev. B 30, 7320 (R) (1984).

[35] M.R. Peterson and C. Nayak, Phys. Rev. Lett. 113, 086401 (2014).

[36] F.D.M. Haldane, Phys. Rev. Lett. 107, 116801 (2011).

[37] R.-Z. Qiu, F.D.M. Haldane, X. Wan, K. Yang and S. Yi, Phys. Rev. B 85, 115308 (2012).

[38] B. Yang, Z. Papić, E.H. Rezayi, R.N. Bhatt and F.D.M. Haldane, Phys. Rev. B 85, 165318 (2012).

[39] V.M. Apalkov and T. Chakraborty, Solid State Commun. 177, 128 (2014).

[40] C.F. Hirjibehedin, I. Dujovne, A. Pinczuk, B.S. Dennis, L.N. Pfeiffer and K.W. West, Phys. Rev. Lett. 95, 066803 (2005).

[41] I.V. Tokatly and G. Vignale, Phys. Rev. Lett. 98, 026805 (2007).

[42] J. Xia, J.P. Eisenstein, L.N. Pfeiffer and K.W. West, Nat. Phys. 7, 845 (2011).

[43] T. Chakraborty and P. Pietiläinen, Phys. Rev. B 39, 7971 (1989); V. Halonen, P. Pietiläinen and T. Chakraborty, Phys. Rev. B 41, 10202 (1990).

[44] Z. Papić, Phys. Rev. B 87, 245315 (2013). 